\title{
An agarose-based microfluidic platform with a gradient buffer for 3D chemotaxis studies
}

\author{
Ulrike Haessler • Yevgeniy Kalinin • Melody A. Swartz • \\ Mingming Wu
}

Published online: 3 April 2009

(C) Springer Science + Business Media, LLC 2009

\begin{abstract}
The current state-of-art in 3D microfluidic chemotaxis device $(\mu \mathrm{FCD})$ is limited by the inherent coupling of the fluid flow and chemical concentration gradients. Here, we present an agarose-based $3 \mathrm{D} \mu \mathrm{FCD}$ that decouples these two important parameters, in that the flow control channels are separated from the cell compartment by an agarose gel wall. This decoupling is enabled by the transport property of the agarose gel, which - in contrast to the conventional microfabrication material such as polydimethylsiloxane (PDMS) - provides an adequate physical barrier for convective fluid flow while at the same time readily allowing protein diffusion. We demonstrate that in
\end{abstract}

Ulrike Haessler and Yevgeniy Kalinin have equal contribution.

Electronic Supplementary Material The online version of this article (doi:10.1007/s10544-009-9299-3) contains supplementary material, which is available to authorized users.

U. Haessler · M. A. Swartz

Institute of Bioengineering, School of Life Sciences,

École Polytechnique Fédérale de Lausanne (EPFL),

1015 Lausanne, Switzerland

M. A. Swartz

Institute of Chemical Sciences and Engineering,

School of Basic Sciences,

École Polytechnique Fédérale de Lausanne (EPFL),

1015 Lausanne, Switzerland

Y. Kalinin $\cdot$ M. Wu

School of Chemical and Biomolecular Engineering,

Cornell University,

Ithaca, NY 14853, USA

M. Wu $(\bowtie)$

Sibley School of Mechanical and Aerospace Engineering,

Cornell University,

Ithaca, NY 14853, USA

e-mail: mw272@cornell.edu this device, a gradient can be pre-established in an agarose layer above the cell compartment (a gradient buffer) before adding the 3D cell-containing matrix, and the dextran $(10 \mathrm{kDa})$ concentration gradients can be re-established within 10 min across the cell-containing matrix and remain stable indefinitely. We successfully quantified the chemotactic response of murine dendritic cells to a gradient of CCL19, an $8.8 \mathrm{kDa}$ lymphoid chemokine, within a type I collagen matrix. This model system is easy to set up, highly reproducible, and will benefit research on 3D chemoinvasion studies, for example with cancer cells or immune cells. Because of its gradient buffering capacity, it is particularly suitable for studying rapidly migrating cells like mature dendritic cells and neutrophils.

Keyword Microfluidics · Cell motility · Chemotaxis . Extracellular matrix

\section{Introduction}

Chemotaxis, or cytokine-directed cell migration, is a wellorchestrated event in which cells move along a chemokine gradient. It is involved in a number of important processes including immune responses, tissue repair, and tumor cell metastasis, and understanding the molecular mechanisms governing directed cell migration is important for the development of new therapeutic strategies. Even though most, if not all, physiological and pathophysiological chemotaxis occurs within $3 \mathrm{D}$ environments, most in vitro chemotaxis experiments have been conducted in 2D settings (Abhyankar et al. 2006; Boyden 1962; Frevert et al. 2006; Irimia et al. 2006; Irimia et al. 2007; Jiang et al. 2005; Li Jeon et al. 2002; Shamloo et al. 2008; Diao et al. 2006; Cheng et al. 2007; Sun et al. 2008; Zigmond 1977). 
In 2D, cell adhesion and detachment to the substrate governs 2D migration. In a 3D microenvironment, cells need to either degrade the extracellular matrix (ECM) or squeeze through small pores in amoeboid fashion (Wolf and Friedl 2008), and they may experience chemokine gradients differently, since many are matrix-binding (Patel et al. 2001). As a result, cells behave very differently in $3 \mathrm{D}$ vs. 2D environments (Behnsen et al. 2007; Cukierman et al. 2001; Griffith and Swartz 2006; Pedersen and Swartz 2005). Thus, to examine cell migration in a physiologically realistic setting, migration assays need to accurately recapitulate the $3 \mathrm{D}$ microenvironment.

To date, most cell migration experiments in $3 \mathrm{D}$ with live cell imaging are performed in open multiwell systems, chamber slides (Zaman et al. 2006), or custom-made sealed chambers (Friedl et al. 1997; Gunzer et al. 1997; Wolf et al. 2007) which do not readily permit the application of welldefined external chemical gradients. Modified Boyden chambers for 3D cell invasion (Semino et al. 2006; Shields et al. 2007) are not conducive to live cell imaging and therefore key parameters like migration speed, persistence ratio, and percentage of migratory cells remain largely unexplored. Recognizing the importance of visualizing cell migration processes in 3D space and time, several microfluidic 3D gradient generation devices have recently been developed (Abhyankar et al. 2008; Mosadegh et al. 2007; Vickerman et al. 2008). All the existing 3D microfluidic chemotaxis devices ( $\mu$ FCDs) are made of PDMS, and are diffusion based, which requires the cell-matrix to be in direct contact with the fluid flow in control channels. This feature complicates the cell seeding protocol. In addition, it is difficult to completely eliminate fluid flow in the cellcontaining matrix since collagen gel (concentration $3 \mathrm{mg} / \mathrm{mL}$ or less) does not provide a reliable barrier for convective flow due to its low hydrodynamic conductivity (Pedersen et al. 2007). This later feature is problematic for cell migration assay, because it is known that even very slow flow can influence cell migratory behavior dramatically (Irimia et al. 2007; Shields et al. 2007).

We present here an agarose-based $3 \mathrm{D} \mu \mathrm{FCD}$, in which the flow control unit is separated from the cell-containing matrix by an agarose gel wall. This feature is a direct consequence of the special transport property of agarose gel. Similar to PDMS, the agarose gel provides an adequate barrier for fluid flow and a stable scaffold for commonly used extracellular matrix (ECM) like fibrin and collagen, but in contrast to PDMS, agarose is a highly hydrated ECM and thus gases, nutrients, and chemokines are readily diffusible. This feature is important for the establishment of a chemical gradient across the ECM with stability and reproducibility, because even very slow flows can alter the chemical distribution (Irimia et al. 2007; Shields et al. 2007). In addition, the agarose gel above the cell compartment creates a "gradient buffer" that can rapidly regenerate a gradient when the chemotaxis channel is filled with the cell-containing matrix. Finally, the thermal gelation feature of the agarose makes the device quick and easy to assemble, and since it does not require unconventional tools such as a plasma cleaner, it is more adaptable to biology labs than a PDMS device.

\section{Materials and methods}

\subsection{Microfluidic device design}

The device is modified from our previously reported 2D $\mu F C D$ (Cheng et al. 2007) and characterized in 3D. It consists of a $1.0 \mathrm{~mm}$ thick agarose membrane patterned with four sets of three-channel units as shown in Fig. 1(a); each three-channel unit is a functional device containing a cell-ECM channel (E) and two flow channels (S1 and S2) (Fig 1(b)). The membrane is sandwiched between a Plexiglas manifold and a stainless steel frame. All the

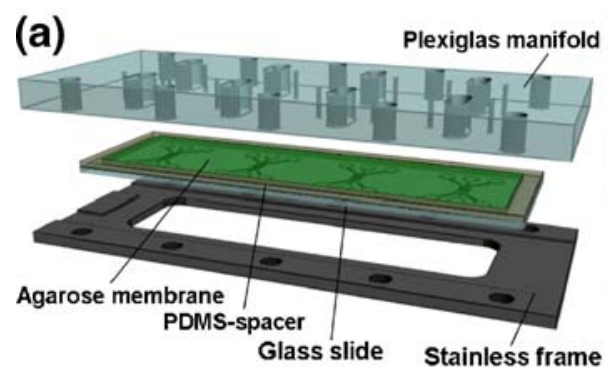

Fig. 1 The 3D microfluidic chemotaxis device design (a) Device schematics. The core of the device is an agarose gel membrane patterned with four three-channel units. The agarose membrane is framed by a PDMS spacer ( $1 \mathrm{~mm}$ thickness), placed on a glass slide and sandwiched between a Plexiglas manifold and a stainless steel frame. (b) Details of a three channel unit. All three channels are

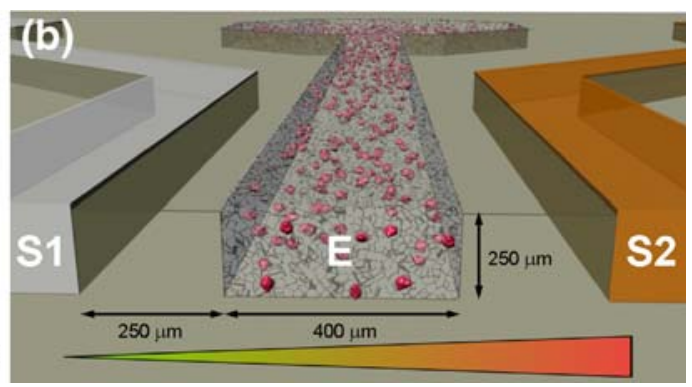

$400 \mu \mathrm{m}$ wide and $250 \mu \mathrm{m}$ deep (The figure is not drawn to scale). Cells are injected together with collagen into the center channel (E), and are embedded in a 3D matrix after the collagen is polymerized. A chemical gradient is generated in the center channel by pumping media containing different concentrations of chemokine through the two side channels (S1 and S2) 
inputs and outputs of the microfluidic channels are machined on the Plexiglas manifold. Each of the three channels are $400 \mu \mathrm{m}$ wide and $250 \mu \mathrm{m}$ high, and the distance between channels is $250 \mu \mathrm{m}$. Gel loading tips were used to connect the inlets and outlets with the tubings. The cells and the collagen mixture were injected into the center channel and the chemokine at different concentrations was pumped through the two side channels at a rate of $5 \mu \mathrm{L} / \mathrm{min}$ in order to maintain fixed chemokine concentrations in the two side channels. Since the chemokine can diffuse through the agarose barrier, a steady linear chemokine gradient is readily established across the center channel (see Fig. 2).

\subsection{Silicon master fabrication}

The silicon master with the positive relief features was fabricated using the standard photolithography technique performed at the Cornell NanoScale Science and Technology Facility. Channel patterns, prepared using CAD software (L-Edit, Tanner Research, Inc.), were printed on chrome-coated glass masks using an optical pattern generator (GCA/MANN 3600F, D.W. Mann/GCA Corp.) capable of $\sim 2 \mu \mathrm{m}$ lateral resolution. Si wafers $(100 \mathrm{~mm}$ diameter, $525 \mu \mathrm{m}$ thick, $<100>$ orientation) were primed (Shipley P20, Rohm and Haas Company) and then spin-coated at 4,000 rpm with photoresist (Megaposit SPR220-7.0, Rohm

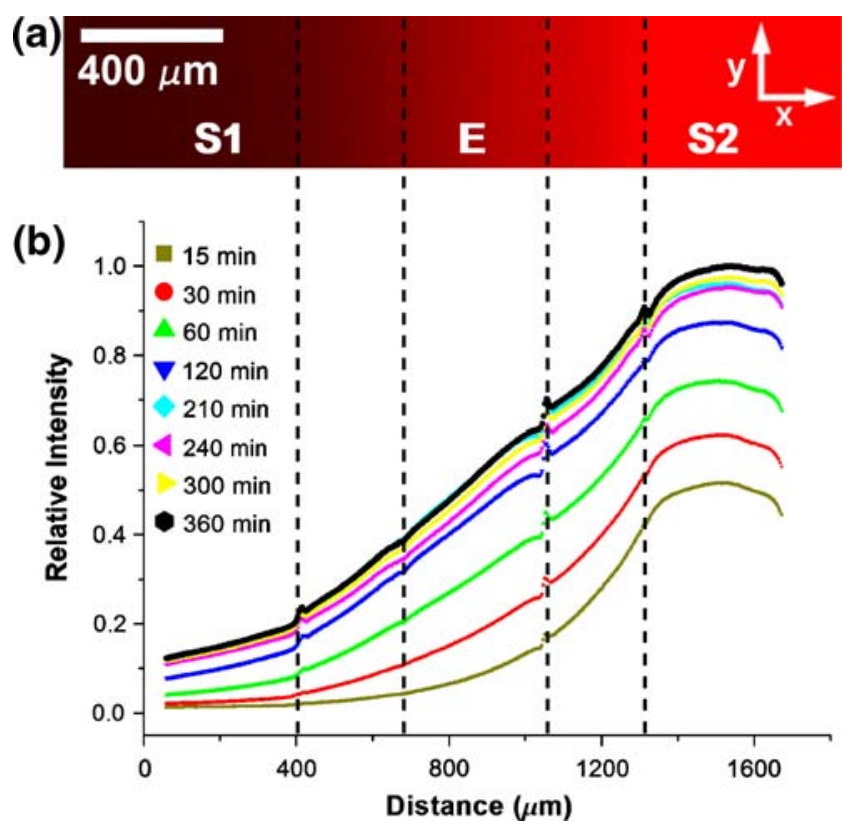

Fig. 2 Device calibration using FITC dextran (a) A fluorescent image of all three channels at a steady state $t=205 \mathrm{~min}$. Here $t=0$ is set when FITC dextran/buffer start to flow in S2/S1 respectively; (b) Time evolution of the FITC dextran concentration profiles across all three channels. It takes about $2 \mathrm{~h}$ for the concentration profile to reach a steady state. The gradient can be kept as long as liquid is pumped through the side channels (here up to $6 \mathrm{~h}$ ) and Haas Company) for $30 \mathrm{~s}$. The resulting film had a thickness of 6-7 $\mu \mathrm{m}$. Spin-coated wafers were baked for $90 \mathrm{~s}$ on a $115 \mathrm{C}$ hotplate, allowed to air-cool, and exposed to broadband UV (365-405 nm) through the mask held in soft contact with a contact aligner (EV620, Electronic Visions Inc.) for $80 \mathrm{~s}$ to a final dose of $\sim 100 \mathrm{~mJ} / \mathrm{cm}^{2}$ in continuous exposure mode. Following a 120 min hold time, the wafer was post-baked at $115 \mathrm{C}$ for $90 \mathrm{~s}$ and then developed for $2 \mathrm{~min}$ (AZ $300 \mathrm{MIF}$, AZ Electronic Materials) using a double-puddle process (HamaTech-Steag wafer processor, HamaTech AG). The wafer was etched for 810 cycles in an inductively coupled plasma reactive ion etcher (Unaxis 770, Plasma-Therm, Inc.). The residual photoresist was later removed by acetone and subsequently stripped by oxygen plasma etching (GaSonics Aura 1000 Asher, Gasonics, Inc.). The resulting step heights were characterized using a profilometer (Tencor P10, KLATencor, Inc.) and found to range from $224 \mu \mathrm{m}$ in the center to $262 \mu \mathrm{m}$ at the perimeter of the etched area with the average height at 240-250 $\mu \mathrm{m}$. The PDMS spacer (Sylgard 184, Dow Corning, Wiesbaden, Germany) was molded on a flat surface and cut out to fit on a commercially available microscope slide ( 1 in. $\times 3$ in.) and surround the chip's structures.

\subsection{Computational modeling of gradient evolution}

The chemical concentration field within our device is computed using a multi-physics modeling software COMSOL (COMSOL Inc., Burlington, MA). In this analysis, we used a computation box of $20 \mathrm{~mm} \times 1 \mathrm{~mm}$ (See Fig. 3(a)). We assume that the normalized chemical concentration $\mathrm{C}$, within the two side channels (S1) and (S2) are fixed at $C=0$ and 1 respectively, and the entire system is governed by a time dependent diffusion equation. The chemical flux at the top/bottom of the device is defined as 0 , that is $\partial C /\left.\partial z\right|_{z=0,1 \mathrm{~mm}}=0$; while the chemical concentration at the lateral boundaries $\left.C\right|_{x= \pm 10 \mathrm{~mm}}=0$. The lateral boundary condition is determined based on the fact that the adjacent device is $\sim 20 \mathrm{~mm}$ away, and the device is primed with buffers before each experiment. The time for chemical (Dextran $10.5 \mathrm{kDa}$ ) to diffuse to a distance of $10 \mathrm{~mm}$ away is about $200 \mathrm{~h}$. We also assume that the diffusion coefficient of the chemical in water and in gel is the same (supported by the linear nature of the steady fluorescent concentration profile across the agarose wall and the media in Fig. 2(b)).

\subsection{Cell culture and ECM preparation}

Murine dendritic cells (mDCs) were derived from bone marrow as described elsewhere (Inaba et al. 1992) and cultured in RPMI 1640 containing 5\% FBS, 1\% antibiotic- 

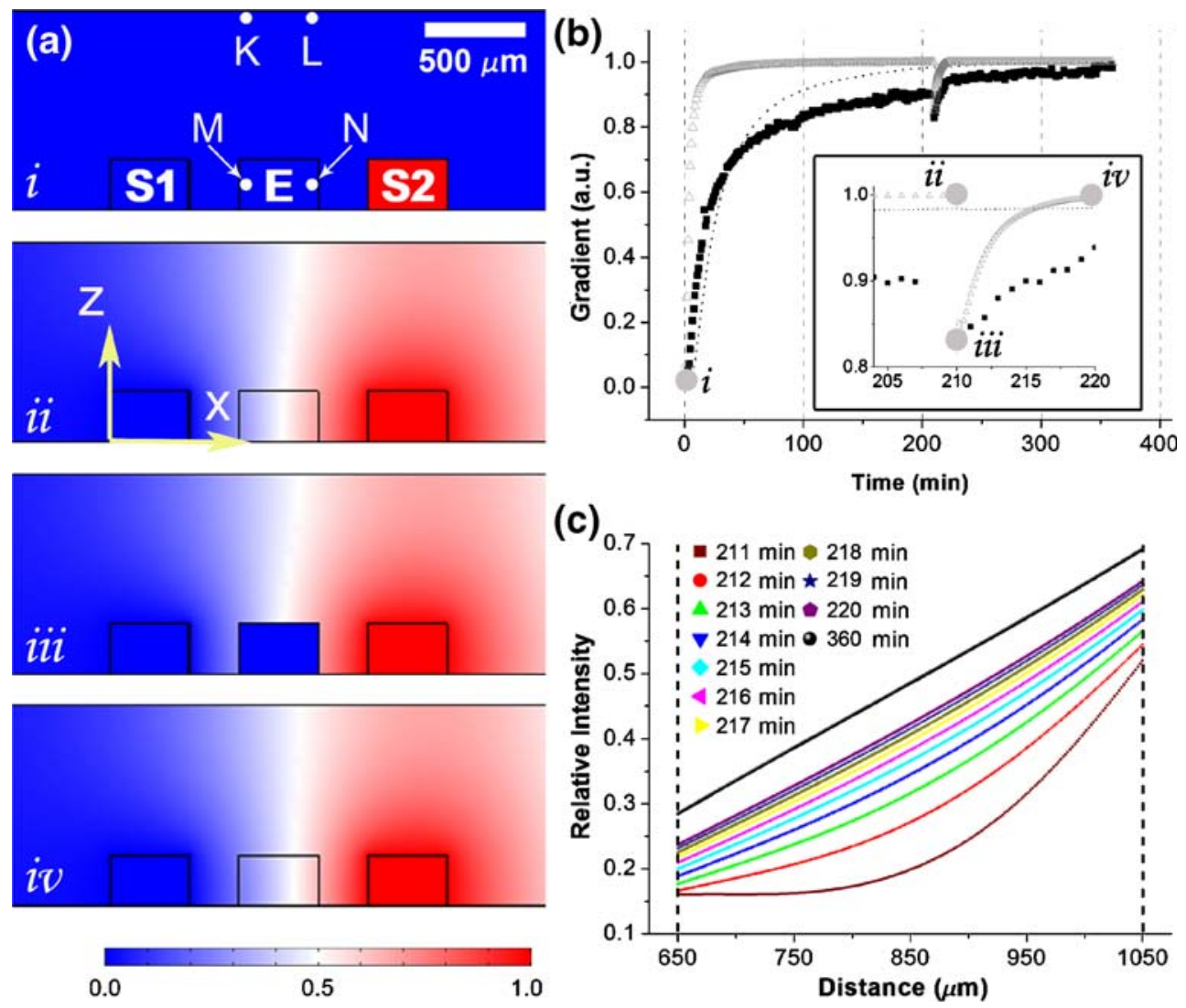

Fig. 3 Validation using COMSOL computation (a) Computed concentration distribution in $\mathrm{x}-\mathrm{Z}$ plane at various time points. Note that the gel thickness is $1 \mathrm{~mm}$ and the channel depth is $250 \mu \mathrm{m}$. (i) At $t=0$, fluids with respective normalized chemical concentrations $C=1$ and $C=0$ start flowing in side channels S1 and S2 respectively; (ii) At $t=205 \mathrm{~min}$, the chemical concentration field reaches steady state; (iii) At $t=210 \mathrm{~min}$, cell-ECM is injected into the center channel (E). Note the change of the chemical concentration in the center channel, but the chemical concentration distribution around the cell compartment remains unchanged due to the buffering capability of the agarose

gel. (iv) At $t=220$ minutes, the chemical concentration gradient in the center channel is re-established. (b) Chemical concentration gradients in the mid of the center channel $(\Delta$, COMSOL) and in the gel above the channel (thin dashed line, COMSOL) are compared with experimental data (-) for a $1.0 \mathrm{~mm}$ thick gel membrane. The insert shows detailed concentration gradient profile right after the refill of the cell-ECM. (c) Time evolution of the chemical concentration profile across the center channel in the mid plane of the center channel (along the line connecting points $\mathrm{M}$ and $\mathrm{N}$ in Fig. 3(a)(i)) right after the re-fill of the cell-ECM in the center channel

antimycotic (all from Gibco, Basel, Switzerland) and 0.5\% supernatant of J558L cells overexpressing mGM-CSF for 6-8 days. They were matured in $500 \mathrm{ng} / \mathrm{ml}$ LPS overnight before seeding into the chamber.

Type I collagen (BD, Basel, Switzerland) was mixed with Matrigel (BD) and mDCs for final concentrations of $1.5 \mathrm{mg} / \mathrm{ml}$ collagen, $10 \%$ matrigel and $10^{6}$ cells $/ \mathrm{ml}$, which was added to each chamber.

\subsection{Agarose-based microfluidic device assembly}

The PDMS spacer of $1 \mathrm{~mm}$ thickness was first placed on the silicon master surrounding four units of threechannel device. 3\% agarose (Promega AG, Dübendorf, Switzerland) in HBSS-buffer (Gibco) was melted and poured into the PDMS-spacer. A microscope slide was immediately placed on top until the agarose polymer- ized. The entire sandwich, consisting of the glass slide, PDMS spacer and agarose membrane, was soaked in media for $30 \mathrm{~min}$ and then assembled as shown in Fig. 1(a). A more detailed description of this procedure can be found in Ref. (Cheng et al. 2007).

\subsection{D cell migration experiments}

All experiments were performed in RPMI 1640 medium containing $10 \mathrm{mM}$ HEPES and $1 \%$ antibiotic-antimycotic, and in some cases complemented with the chemokine CCL19 (R\&D Systems, Abingdon, United Kingdom). The assembled chamber was cooled on ice before injecting the cell-containing ECM to facilitate a homogenous polymerization of the gel after injection into the device (data not shown). Gradients were pre-established by pumping medium, alone or supplemented with CCL19, through the side 
channels for $2 \mathrm{~h}$ with the device on ice. Then, $\sim 30 \mu \mathrm{l}$ of the cell-ECM mixture was injected into each of the four center channels and both inlets and outlets were sealed with PDMS plugs. The entire device was transferred onto the $37 \mathrm{C}, 5 \% \mathrm{CO}_{2}$ incubated microscope stage and turned upside-down to avoid cell settling. After $10 \mathrm{~min}$, the device was turned again into the upright position and an additional $20 \mathrm{~min}$ were allowed for collagen polymerization before imaging began.

\subsection{Imaging and data analysis}

Images were taken in bright field on an inverted fluorescent microscope (Zeiss Axiovert $200 \mathrm{M}$, Switzerland) with a CCD camera (AxioCam MRm) at 1-min intervals for $\sim 2 \mathrm{~h}$. Images were then processed off-line using ImageJ (NIH, Bethesda, $\mathrm{MD)}$ to track cells and Matlab to analyze the data. Cells were considered migratory if they moved more than one cell diameter within one hour. Migratory speed was calculated as the average incremental speed at $1 \mathrm{~min}$ intervals. Persistence length for each cell was calculated as the net distance divided by the length of the trajectory, and migratory speed parallel to gradient was defined as the total distance travelled parallel to the applied gradient divided by time.

\section{$2.83 \mathrm{D}$ image reconstruction}

A z-stack of 50 images with a $5 \mu \mathrm{m}$ step size was taken in phase contrast and converted into a 3D image using Imaris software (Bitplane, Zurich, Switzerland). A Gaussian filter was applied to the red channel and overlaid with the original green and blue channels. Cells were found automatically, and then $10.2 \mu \mathrm{m}$ diameter spheres representing the cells were overlaid with the original image.

\section{Results and discussions}

\subsection{Device characterization}

We used FITC labeled dextran $(10 \mathrm{kDa})$ to visualize the gradient formation and numerical simulation in COMSOL to estimate the diffusion kinetics. In both methods, we demonstrated that after a chemical gradient is established, it can be re-establisehed upon filling the center compartment with the cell-ECM within $10 \mathrm{~min}$.

Gradient characterization using FITC dextran To visualize the gradient, $10 \mathrm{kDa}$ FITC-dextran (Sigma-Aldrich) was used, which is close to the molecular weight of CCL19 $(8.8 \mathrm{kDa})$. All three channels were first primed with cell culture medium prior to experiments. At time $t=0,0.1 \mathrm{mM}$ FITC-dextran in cell culture medium and blank medium were pumped through the two side channels (S2) and (S1) respectively; at $t=210 \mathrm{~min}$, cell-ECM was injected in the center channel (E). A time series of images of all three channels was taken at 1-minute intervals as shown in Fig. 2(b). The initial time evolution of the fluorescence intensity profiles across all three channels occured within approximately $120 \mathrm{~min}$; i.e., it took $\sim 2 \mathrm{~h}$ for the concentration gradient to reach steady state (Fig. 2(b)). However, the time for the system to re-establish a steady gradient after refilling the middle chamber with cells and ECM was less than $10 \mathrm{~min}$ (See insert of Fig. 3(b)). It should be noted that the measured fluorescence intensity is not a precise representation of the chemical concentration in the cell-ECM chamber, because the camera records both the fluorescent light emitting from the cell-ECM chamber and the agarose gel above it. For this reason, a detailed calculation of the concentration field of the system was carried out.

Gradient characterization using numerical computation The spatial and temporal chemical concentration profiles within the cell-ECM chamber were computed using COMSOL. The normalized chemical concentrations in the two side channels (S1) and (S2) were fixed at zero and one. We use diffusion coefficient $D=111 \mu \mathrm{m}^{2} / \mathrm{sec}$ (Lebrun and Junter 1993) for dextran of molecular weight of $10.5 \mathrm{kDa}$ diffusing in deionized water at $25 \mathrm{C}$. We considered three events: first, establishing a stable concentration gradient within the entire chamber (Fig. 3(a), i-ii); second, immediately after filling the center compartment with a cell-ECM mixture, where the gradient is disrupted only in this compartment (Fig. 3(a), iii); and finally, re-establishing the stable gradient across the cell-ECM channel (Fig. 3(a), iv). The agarose above the chamber has a buffering effect because it retains the gradient after filling the center compartment and serves as a source for re-establishment along with the side channels.

The time evolution of the concentration gradient in the midplane (shown as M, N in Fig. 3(a)(i)) of the cellECM chamber as well as at two points (shown as K, L in Fig. 3(a)(i)) above the chamber in the agarose gel are plotted in Fig. 3(b). The insert in Fig. 3(b) demonstrates that it takes $\sim 3 \mathrm{~min}$ for the gradient to re-establish within the cell chamber (E) after the cell-ECM injection. Figure 3(c) shows detailed concentration profiles across the center channel after the cell-ECM refill at $t=210 \mathrm{~min}$. It demonstrates that it takes about $10 \mathrm{~min}$ for the overall concentration profile to reach steady state (or within $10 \%$ of its final value). While the results from FITC dextran calibration and the computation are in reasonably good agreement (Fig. 3(b)), the time scale for FITC dextran gradient to establish (or re-establish) in experiments is longer than that of computation. We believe that this is a 
result that the camera is recording fluorescent light emitted from all z-layers of $1 \mathrm{~mm}$ thick agarose gel, while the computation only records concentration at a particular z-plane.

\subsection{CCL19-dependent dendritic cell chemotaxis}

To demonstrate suitability to chemotaxis experiments and biocompatibility, we examined dendritic cell (DC) chemotaxis up a chemokine gradient in a 3D ECM. Mature murine DCs (mDCs) were exposed to a chemokine gradient of $0.11 \mathrm{nM} / \mu \mathrm{m}$ CCL19, created by flowing cell culture medium containing $100 \mathrm{nM}$ CCL19 in one side channel and $0 \mathrm{nM}$ in the other. Assuming a cell diameter of roughly 10-20 $\mu \mathrm{m}$ (depending on how spread they are), the transcellular concentration difference would be on the order of 1-2 nM, with a relative concentration difference percentile (concentration difference across a cell divided by the average concentration) of $\sim 2-4 \%$ in the center of the channel. Using time lapse movies of migrating mDCs, we obtained trajectories for 7-15 cells per chamber for a duration of $2 \mathrm{~h}$ (Fig. 4). Movies of mDCs migrating in various CCL19 concentration gradients can be viewed in the supplemental material (SM1 and SM2). In the absence of chemokine there is no preferential migratory direction (See SM1 and Fig. 4(a, c)). In the presence of a concentration gradient of $0.11 \mathrm{nM} / \mu \mathrm{m}$, directed cell migration is clearly seen in the movie (SM2), the overlay $\mathrm{mDC}$ trajectories and the polar plots (Fig. 4(b, d)).

Using the cell migration trajectories shown in Fig. 4, we evaluated several parameters quantifying the cell motility and the chemotactic response at various chemokine concentrations. We saw, as expected, that mDCs become more motile and directed in their migration in the presence of a CCL19 concentration gradient (Table 1). The percentage of migrating cells, defined by those moving $>1$ cell diameter within one hour, increased in the presence of the gradient, but the overall speed was only slightly enhanced. Their average persistence length, defined by the net distance travelled divided by the total length of the trajectory for each cell, was strongly increased in the presence of the gradient. It should be noted that a detailed examination of DC response to exogenous chemokine gradients, which is important for gaining insight into how DCs integrate chemokine signals, is beyond the scope of this study. This example shows how quantitative analysis for migratory parameters under very well-controlled gradients becomes accessible for highly migratory cells such as DCs chemotacting within a 3D environment.
Fig. 4 Chemotactic responses of murine dendritic cells (mDCs) to chemokine CCL19 concentration gradient (a-b) Polar plots of trajectories of migrating $\mathrm{mDCs}$ in the absence and presence of a chemical concentration gradients; (c-d) Images of cells in the center channel with the overlay cell tracks. Each line is a track of a migrating cell and the time duration of each track is $120 \mathrm{~min}$. The chemokine concentration along the two side channels are $(0: 0)$ for (a) and (c), (0: $100 \mathrm{nM})$ for (b) and (d). The scale bar, $50 \mu \mathrm{m}$ (a)
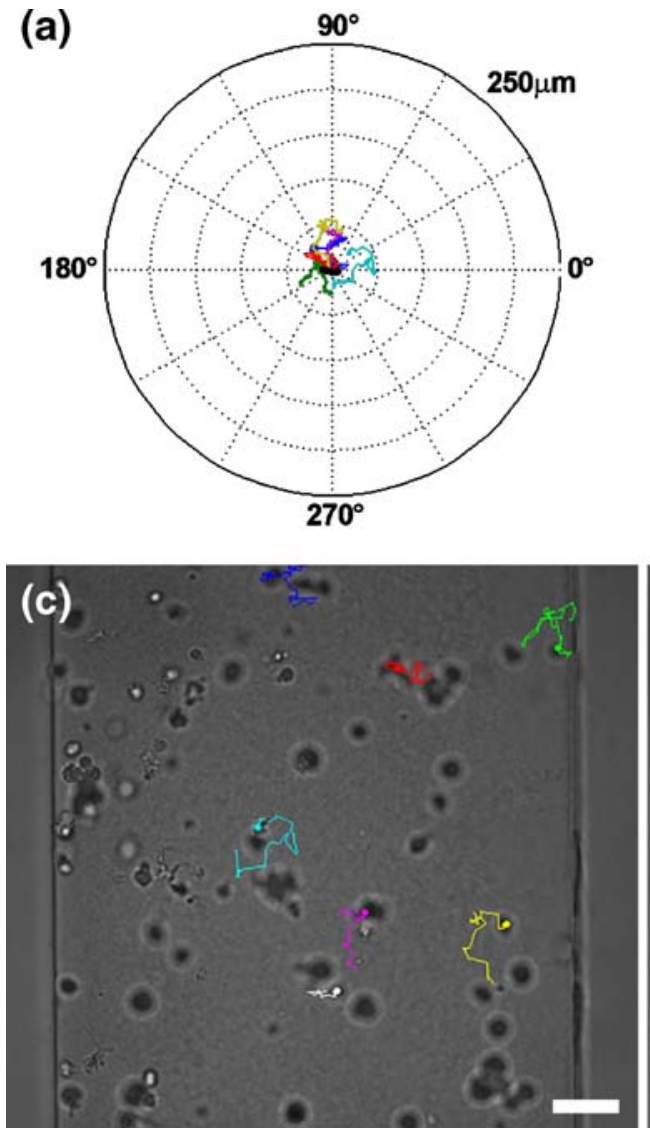

(b)
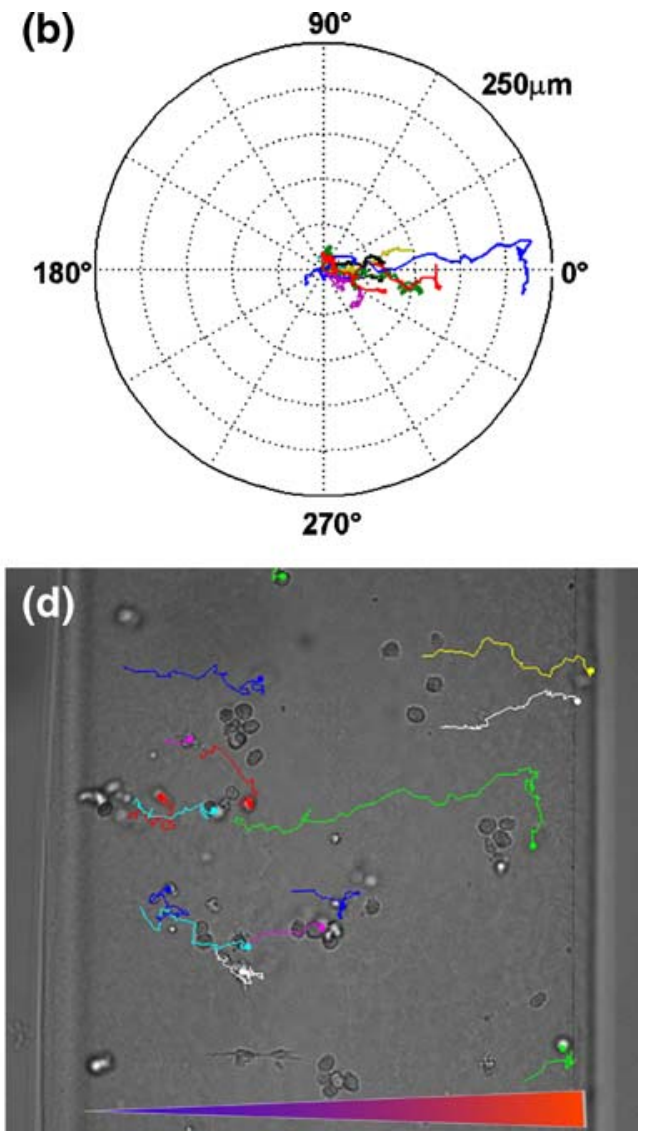
Table 1 Quantitative characterization of chemotactic responses of mouse DCs to CCL19 in one representative set of experiments. Note that the DC migration parameters depend on the state of maturation, tissue source of DCs and isolation method, collagen gel composition, time after isolation, and even strain of mouse (Gunzer et al. 2000); these details are not addressed here

\begin{tabular}{lcccc}
\hline & Percentage cells migrating & Migratory speed $(\mu \mathrm{m} / \mathrm{min})$ & Velocity parallel to gradient & Persistence \\
\hline No gradient & $8.4 \%$ & $1.33( \pm 0.47)$ & $0.004( \pm 0.13)$ & $0.19( \pm 0.09)$ \\
CCL19 $(0: 50 \mathrm{nM})$ & $18.8 \%$ & $1.33( \pm 0.73)$ & $0.26( \pm 0.84)$ & $0.27( \pm 0.24)$ \\
CCL19 $(0: 100 \mathrm{nM})$ & $22.1 \%$ & $1.51( \pm 0.87)$ & $0.55( \pm 0.64)$ & $0.37( \pm 0.19)$ \\
\hline
\end{tabular}

\subsection{Three dimensionality of cell seeding}

Without any gel injected together with the cells, dendritic cells attach to the glass surface and migrate on a $2 \mathrm{D}$ substrate. When injecting the cell-collagen mixture, cells are distributed uniformly in all three dimensions. This three dimensionality is clearly demonstrated in Fig. 5. There are two important improvements that we have made to ensure three-dimensionality of cell seeding. One is by increasing the channel depth from $100 \mu \mathrm{m}$ (in the case of 2D model) to $250 \mu \mathrm{m}$; and the other is by turning the device upside down for $10 \mathrm{~min}$ right after cell seeding and during gel polymerization. Without these two steps, most of the bigger cells sink to the glass-gel interface, and attach to the glass plate and migrate more or less on a $2 \mathrm{D}$ plane. The side views in Fig. 5 are reconstructed images from 3D phase contrast z-stacks using a commercial software package (See the methods section for details) demonstrating that cells are distributed uniformly in $\mathrm{z}$ direction.

\section{Concluding remarks}

We have developed an agarose-based 3D $\mu \mathrm{FCD}$ that allows for the quantitative analysis of migrating cells in a quickly stabilized chemical gradient and within a 3D
ECM. The unique feature of the 3D agarose-based $\mu F C D$ is its ability to decouple the control of fluid flow and chemical gradients within a cell-containing ECM, in contrast to the exiting PMDS-based 3D $\mu \mathrm{FCD}$ models. This decoupling comes from the unique transport property of the agarose gel, in that it allows nutrients and chemicals to diffuse through, but provides an effective barrier for convective flows. We demonstrated that the agarose gel above the cell-compartment acts as a 'buffer', which allows us to pre-establish a gradient, and then seed the cells. The buffering capability also enables us to re-establish a chemical gradient of dextran $(10 \mathrm{kDa})$ across a $400 \mu \mathrm{m}$ wide cell-ECM channel within $10 \mathrm{~min}$. This is important, especially for those cells (e.g. immune cells) that migrate in a time scale comparable to the gradient establishing time.

It is known that the fluid flow and chemical gradients are two key parameters that define the cell microenvironment. The ability to decouple these two parameters opens door for studies of mechanical cues (shear stresses) and chemical cues individually and together. It also allows for studies such as auto/paracrine signalling, and cell-cell interactions.

The $3 \mathrm{D} \mu \mathrm{FCD}$ presented here can be easily adapted to study the relative responses of cells to dual gradients, mimicking a complex chemical environment that is commonly seen in nature. One immediate interest for us would
Fig. 5 Three dimensionality of murine dendritic cells in 3D $\mu \mathrm{FCD}$ device Dendritic cells were seeded on the plane surface of a 2D chamber slide (2D views) or in a $3 \mathrm{D}$ collagen-Matrigel matrix (3D views), respectively. Upper row are top views of cells on a $2 \mathrm{D}$ surface and in a 3D matrix. Images are taken using phase contrast. Lower row are the corresponding side views of the cells in 3D matrix, reconstructed using a series of z-slice images. Note, in the presence of a collagen-Matrigel matrix, cells are evenly distributed along the vertical direction, Scale bar, $50 \mu \mathrm{m}$
2D top view

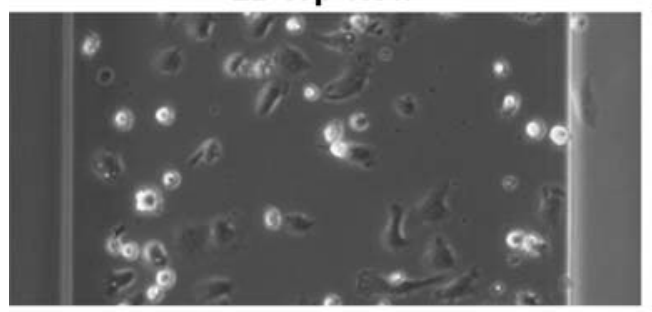

2D side view

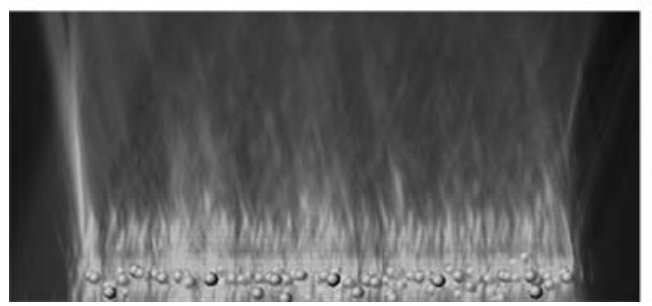

3D top view

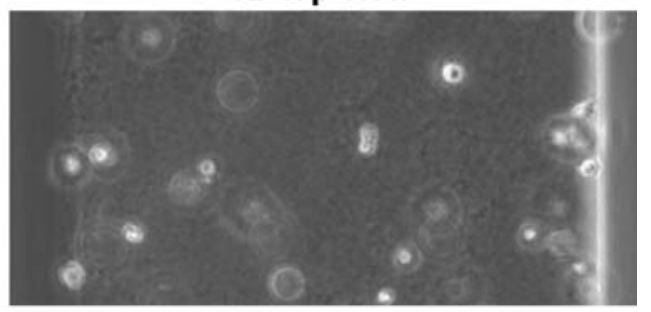

3D side view

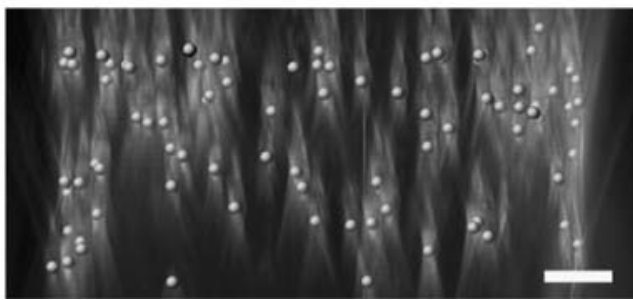


be to test the relative potency of $\mathrm{mDCs}$ to soluble chemokine CCL19 and matrix binding chemokine CCL21, both are homing chemokines to lymphatics. Quantification of dendritic cell chemotactic behavior in a complex chemical environment will provide vital information for developing novel medical devices to adjust unbalanced immune responses commonly seen in autoimmune diseases, allergies and implant rejection. It should be noted that other cell types such as cancer cells and stem cells can be readily used in this device for quantitative analysis of cell migration in various chemokine and growth factor gradients.

Acknowledgments MW would like to thank all members of the Swartz lab during her half year visit there, MW and YK acknowledge the very helpful technical support from Andrew Darling and Nak Won Choi. MW and YK were supported by funds from the National Science Foundation (CBET-0619626) and by grants from the Nanobiotechnology Center (NBTC), an STC Program of the National Science Foundation under Agreement No. ECS-9876771. MAS and UH were supported by the Swiss National Science Foundation (107602 and 310010).

\section{References}

V.V. Abhyankar, M.A. Lokuta, A. Huttenlocher, D.J. Beebe, Characterization of a membrane-based gradient generator for use in cellsignaling studies. Lab Chip 6(3), 389-393 (2006). doi:10.1039/ b514133h

V.V. Abhyankar, M.W. Toepke, C.L. Cortesio, M.A. Lokuta, A. Huttenlocher, D.J. Beebe, A platform for assessing chemotactic migration within a spatiotemporally defined 3D microenvironment. Lab Chip 8(9), 1507-1515 (2008). doi:10.1039/b803533d

J. Behnsen, P. Narang, M. Hasenberg, F. Gunzer, U. Bilitewski, N. Klippel, M. Rohde, M. Brock, A.A. Brakhage, M. Gunzer, Environmental dimensionality controls the interaction of phagocytes with the pathogenic fungi Aspergillus fumigatus and Candida albicans. PLoS Pathog 3(2), e13 (2007). doi:10.1371/ journal.ppat.0030013

S. Boyden, The chemotactic effect of mixtures of antibody and antigen on polymorphonuclear leucocytes. J. Exp. Med. 115, 453-466 (1962). doi:10.1084/jem.115.3.453

S.Y. Cheng, S. Heilman, M. Wasserman, S. Archer, M.L. Shuler, M. $\mathrm{Wu}$, A hydrogel-based microfluidic device for the studies of directed cell migration. Lab Chip 7(6), 763-769 (2007). doi:10.1039/b618463d

E. Cukierman, R. Pankov, D.R. Stevens, K.M. Yamada, Taking cellmatrix adhesions to the third dimension. Science 294(5547), 1708-1712 (2001). doi:10.1126/science.1064829

J. Diao, L. Young, S. Kim, E.A. Fogarty, S.M. Heilman, P. Zhou, M.L. Shuler, M. Wu, M.P. DeLisa, A three-channel microfluidic device for generating static linear gradients and its application to the quantitative analysis of bacterial chemotaxis. Lab Chip 6(3), 381-388 (2006). doi:10.1039/b511958h

C.W. Frevert, G. Boggy, T.M. Keenan, A. Folch, Measurement of cell migration in response to an evolving radial chemokine gradient triggered by a microvalve. Lab Chip 6(7), 849-856 (2006). doi:10.1039/b515560f

P. Friedl, K. Maaser, C.E. Klein, B. Niggemann, G. Krohne, K.S. Zanker, Migration of highly aggressive MV3 melanoma cells in 3-dimensional collagen lattices results in local matrix reorganization and shedding of alpha2 and beta1 integrins and CD44. Cancer Res. 57(10), 2061-2070 (1997)

L.G. Griffith, M.A. Swartz, Capturing complex 3D tissue physiology in vitro. Nat. Rev. Mol. Cell Biol. 7(3), 211-224 (2006). doi:10.1038/nrm1858

M. Gunzer, E. Kampgen, E.B. Brocker, K.S. Zanker, P. Friedl, Migration of dendritic cells in 3D-collagen lattices. Visualisation of dynamic interactions with the substratum and the distribution of surface structures via a novel confocal reflection imaging technique. Adv. Exp. Med. Biol. 417, 97-103 (1997)

M. Gunzer, P. Friedl, B. Niggemann, E.B. Broker, E. Kampgen, K.S. Zanker, Migration of dendritic cells within 3-D collagen lattices is dependent on tissue origin, state of maturation, and matrix structure and is maintained by proinflammatory cytokines. J. Leukoc. Biol. 67(5), 622-629 (2000).

K. Inaba, M. Inaba, N. Romani, H. Aya, M. Deguchi, S. Ikehara, S. Muramatsu, R.M. Steinman, Generation of large numbers of dendritic cells from mouse bone marrow cultures supplemented with granulocyte/macrophage colony-stimulating factor. J. Exp. Med. 176(6), 1693-1702 (1992). doi:10.1084/jem.176.6.1693

D. Irimia, S.Y. Liu, W.G. Tharp, A. Samadani, M. Toner, M.C. Poznansky, Microfluidic system for measuring neutrophil migratory responses to fast switches of chemical gradients. Lab Chip 6 (2), 191-198 (2006). doi:10.1039/b511877h

D. Irimia, G. Charras, N. Agrawal, T. Mitchison, M. Toner, Polar stimulation and constrained cell migration in microfluidic channels. Lab Chip 7(12), 1783-1790 (2007). doi:10.1039/ b710524j

X. Jiang, Q. Xu, S.K. Dertinger, A.D. Stroock, T.M. Fu, G.M. Whitesides, A general method for patterning gradients of biomolecules on surfaces using microfluidic networks. Anal. Chem. 77(8), 2338-2347 (2005). doi:10.1021/ac048440m

L. Lebrun, G.A. Junter, Diffusion of sucrose and dextran through agar gel membranes. Enzyme Microb. Technol. 15(12), 1057-1062 (1993). doi:10.1016/0141-0229(93)90054-6

N. Li Jeon, H. Baskaran, S.K. Dertinger, G.M. Whitesides, L. Van de Water, M. Toner, Neutrophil chemotaxis in linear and complex gradients of interleukin- 8 formed in a microfabricated device. Nat. Biotechnol. 20(8), 826-830 (2002)

B. Mosadegh, C. Huang, J.W. Park, H.S. Shin, B.G. Chung, S.K. Hwang, K.H. Lee, H.J. Kim, J. Brody, N.L. Jeon, Generation of stable complex gradients across two-dimensional surfaces and three-dimensional gels. Langmuir 23(22), 10910-10912 (2007). doi:10.1021/la7026835

D.D. Patel, W. Koopmann, T. Imai, L.P. Whichard, O. Yoshie, M.S. Krangel, Chemokines have diverse abilities to form solid phase gradients. Clin. Immunol. 99(1), 43-52 (2001). doi:10.1006/ clim.2000.4997

J.A. Pedersen, M.A. Swartz, Mechanobiology in the third dimension. Ann. Biomed. Eng. 33(11), 1469-1490 (2005). doi:10.1007/ s10439-005-8159-4

J.A. Pedersen, F. Boschetti, M.A. Swartz, Effects of extracellular fiber architecture on cell membrane shear stress in a 3D fibrous matrix. J. Biomech. 40(7), 1484-1492 (2007). doi:10.1016/j.jbio mech.2006.06.023

C.E. Semino, R.D. Kamm, D.A. Lauffenburger, Autocrine EGF receptor activation mediates endothelial cell migration and vascular morphogenesis induced by VEGF under interstitial flow. Exp. Cell Res. 312(3), 289-298 (2006)

A. Shamloo, N. Ma, M.M. Poo, L.L. Sohn, S.C. Heilshorn, Endothelial cell polarization and chemotaxis in a microfluidic device. Lab Chip 8(8), 1292-1299 (2008). doi:10.1039/b719788h

J.D. Shields, M.E. Fleury, C. Yong, A.A. Tomei, G.J. Randolph, M.A. Swartz, Autologous chemotaxis as a mechanism of tumor cell homing to lymphatics via interstitial flow and autocrine CCR7 
signaling. Cancer Cell 11(6), 526-538 (2007). doi:10.1016/j. ccr.2007.04.020

K. Sun, Z. Wang, X. Jiang, Modular microfluidics for gradient generation. Lab Chip, 2008

V. Vickerman, J. Blundo, S. Chung, R. Kamm, Design, fabrication and implementation of a novel multi-parameter control microfluidic platform for three-dimensional cell culture and real-time imaging. Lab Chip 8(9), 1468-1477 (2008). doi:10.1039/b802395f

K. Wolf, P. Friedl, Mapping proteolytic cancer cell-extracellular matrix interfaces. Clin Exp Metastasis, 2008

K. Wolf, Y.I. Wu, Y. Liu, J. Geiger, E. Tam, C. Overall, M.S. Stack, P. Friedl, Multi-step pericellular proteolysis controls the transition from individual to collective cancer cell invasion. Nat. Cell Biol. 9(8), 893-904 (2007). doi:10.1038/ ncb1616

M.H. Zaman, L.M. Trapani, A. Siemeski, D. Mackellar, H. Gong, R.D. Kamm, A. Wells, D.A. Lauffenburger, P. Matsudaira, Migration of tumor cells in 3D matrices is governed by matrix stiffness along with cell-matrix adhesion and proteolysis. Proc. Natl. Acad. Sci. USA 103(29), 10889-10894 (2006). doi:10.1073/pnas.0604460103

S.H. Zigmond, Ability of polymorphonuclear leukocytes to orient in gradients of chemotactic factors. J. Cell Biol. 75(2 Pt 1), 606-616 (1977). doi:10.1083/jcb.75.2.606 